\title{
Frame structure damage identification based on wavelet - artificial
}

\section{immune algorithm}

\author{
Deqing Guan ${ }^{1, a}$, Jiawei Zhang ${ }^{1, b}$, Xiong Tang ${ }^{1, c}$ \\ ${ }^{1}$ Changsha University of Science \& Technology, College of civil engineering and architecture, \\ Hunan, Changsha \\ a491596452@qq.com, b570716904@qq.com, ‘876812458@qq.com
}

\begin{abstract}
Keywords: damage, wavelet analysis, artificial immune algorithm, simple frame structure Abstract. Structural damage detection and diagnosis is a very important factor of the structural safety. In order to improve the application of artificial intelligence algorithms for structural damage detection, we try to wavelet analysis and artificial immune algorithm to combine the analysis by finite element and Matlab programming. The result of the simulation and verification by analyzing simple frame structure suggest that this approach combines the advantages of wavelet analysis and artificial immune algorithm : quickly and accurately identifying the damage location and calculating the extent of the damage.This method can be used in engineering practice of structural damage detection to improve the efficiency of structural damage identification.
\end{abstract}

\section{Introduction}

Structure in the long-term external factors will inevitably lead to structural damage accumulation, and even catastrophic accidents Therefore, the study of structural damage identification problem has important theoretical significance and engineering application value.

In 1974, the concept of wavelet transform was first proposed by the engineer J.Morlet who was engaged in signal processing of oil in France. This is a new digital signal processing method, with good self-adaptability and local analysis. Hong ${ }^{[1]}$ successfully used the continuous wavelet to identify the damage location of the beam. M.Rucka and K.Wilde ${ }^{[2]}$ ect uses continuous wavelet pairs to damage the cantilever beam and the four-sided plate model. The results show that the corresponding damage location where the wavelet mathematical model arrive at the maximum point. Guan Deqing $^{[3]}$ put forward that the problem of frame structure damage identification of strain mode can be solved by using wavelet transform. Guan Deqing,ect ${ }^{[4]}$ put up with using avelet theory to solve such as elastic plate damage identification. Guan Deqing ${ }^{[5]}$ combined wavelet and genetic algorithm to identify the damage of frame structure.

Artificial Immune Algorithm is a kind of new type of intelligent Algorithm in the structural damage research. Researchers apply concept and theory of immune in the genetic Algorithm (IA), under the premise of keeping the good qualities of original Algorithm, They try to use some characteristics of the information or knowledge of the unsettled problems to suppress the degradation phenomenon appeared in the process of optimization, the Algorithm is called Immune Algorithm ${ }^{[6]}$. Michelle, etc. ${ }^{[7]}$ has studied the bionic mechanism of the artificial immune system to solve the problem of structural damage identification and classification. Hui-yong guo etc. ${ }^{[8]}$ Studies show that the artificial immune algorithm is better than traditional artificial intelligence algorithm in stronger ability of global optimization, when applied to the structural damage diagnosis. 


\section{Wavelets-Principle of Artificial Immune Algorithm}

Detecting Damage Location Based on the Method of Vibration and Modal Wavelets Transform. The continuous wavelets transformation of arbitrary assumptive function $f(t) \in L^{\wedge} 2(R)$ can be expressed as :

$$
W f(a, \tau)=\frac{1}{\sqrt{a}} \int_{-\infty}^{+\infty} f(t) \psi^{*}\left(\frac{t-\tau}{a}\right) d t .
$$

Where $\psi(\mathrm{t})$ is the mother wavelets. a is the scale factor and $\tau$ is the shift factor. Where $\psi^{*}(\mathrm{t})$ is the conjugate function of $\psi(\mathrm{t})$. According to the formula (4), local features of the function can be highlighted by the continuous wavelets transformation both in time domain and in frequency domain. Then the singularity of function is analyzed on the base of the local features.

The damage of structure units can be imitated by the means of reducing the bending rigidity. The structure still satisfy both the compatibility conditions and internal force equilibrium conditions although that $\mathrm{EI}(\mathrm{V}+)$ and $\mathrm{EI}\left(\mathrm{V}_{-}\right)$at each side of damage cross section $\mathrm{V}$ are unequal. Its displacement, corner, bending moment relationship as eq (2),(3),(4).

$$
\begin{aligned}
& \omega\left(v^{+}\right)=\omega\left(v^{-}\right) . \\
& \frac{d \omega\left(v^{+}\right)}{d x}=\frac{d \omega\left(v^{-}\right)}{d x} . \\
& E I\left(v^{+}\right) \frac{d^{2} \omega\left(v^{+}\right)}{d x^{2}}=E I\left(v^{-}\right) \frac{d^{2} \omega\left(v^{-}\right)}{d x^{2}} .
\end{aligned}
$$

Therefore, continuous wavelet transform was carried out on the $\mathrm{w}(\mathrm{x})$ by using the second derivative of Gauss function and wavelets transformation modulus maximum corresponds to the damage location.

The Calculating Principle of Damage Degree Based on the Artificial Immune Algorithm. Artificial immune algorithm is a new algorithm for simulating biological immune system and enabling multi-peak search and global optimization of multi-peak function.

Encoding. In the paper damage degree of damaged units was defined by the antibody which adopts the binary encoding.

Assuring Population Scale. Population scale refers to the number of individuals in population, and in this paper it refers to the number of antibodies. In order to assure the high computational efficiency as well as the diversity of the individuals, the number of individuals is defined as 300 according to the population model.

Affinity Calculation. Affinity between antibody and antigen is the unique combination in the immune system. The calculating approach follows as:

$$
\begin{aligned}
& (A g)_{j}=\frac{1}{1+t_{j}}+\omega_{j} * \operatorname{praise}(j) \\
& \operatorname{praise}(j)=\exp \left(-\frac{1}{\operatorname{fitness}(j)}\right) \\
& \operatorname{fitness}(j)=\frac{1}{1+t_{j}}
\end{aligned}
$$

Where $(\mathrm{Ag})_{\mathrm{j}}$ is the affinity between antigen $\mathrm{Ag}$ and antibody $\mathrm{j}$. Where $\mathrm{w}_{\mathrm{j}}$ is the weight 
coefficient of encouraging function.

The Similarity of Antibody. Affinity probability of antibody Pf is the rotation between the affinity of antibody $i$ and the total affinity of the whole antibodies in the population. It can be expressed as :

$$
P_{f}=\frac{(A g)_{i}}{\sum_{j=1}^{N}(A g)_{j}} \text {. }
$$

In order to select the new generation of antibody, the roulette wheel method is used, and the selection probability is the following expression :

$$
P=\alpha P_{f}+(1-\alpha) P_{d}
$$

Where $\alpha$ is the affinity coefficient which ranges from 0 to 1 .

Operation of Selection, Crossover and Mutation. In the process of operating of selection, crossover and mutation, the antibody between antibody with the highest affinity will be conserved and take the place of the head antibody of the original population. Then use this approach to operate other part of the antibodies to generate new antibodies.

Objective Function. The objective function using the error function of structure frequency and modal shape may be expressed as following:

$$
J=F_{\omega} \sum_{i=1}^{m}\left(\frac{f_{i}^{\text {test }}-f_{i}^{c a l}}{f_{i}^{\text {test }}}\right)^{2}+F_{\Phi} \sum_{i=1}^{n} \sum_{j=1}^{k}\left(\phi_{i j}^{\text {test }}-\phi_{i j}^{c a l}\right)^{2} .
$$

$F_{\omega}$ and $F_{\Phi}$ are weighted factors, $f_{i}^{\text {test }}$ is the vibration frequency of field measurement and $f_{i}^{\text {cal }}$ is the vibration frequency of calculation. Respectively $\phi_{i j}^{\text {test }}$ and $\phi_{i j}^{c a l}$ are the vibration frequency of field measurement and calculation which are normalized. $\mathrm{m}$ is the frequency order of the calculation and evaluation and $\mathrm{k}$ is the number of mode displacement. The smaller the value of $\mathbf{J}$ is, the better the antibody is, and the better the calculation result matches with actual damage degree.

\section{Numerical Simulation Analysis}

This article uses the A-span and two-storey frame to test the validity of the damage identification method for simple structure as previously described.

Overview: material is steel, span is $3 \mathrm{~m}$, the length of the beams and columns are 3 meters .cross-sectional dimensions: $0.2 \times 0.25 \mathrm{~m}^{2}$, elastic modulus $\mathrm{E}=2.07 \times 10^{11}$, density $\rho=7800 \mathrm{~kg} /$ $m^{3}$, Poisson's ratio $\mu=0.3$, the frame is divided into 300 units equidistant by the finite element method.

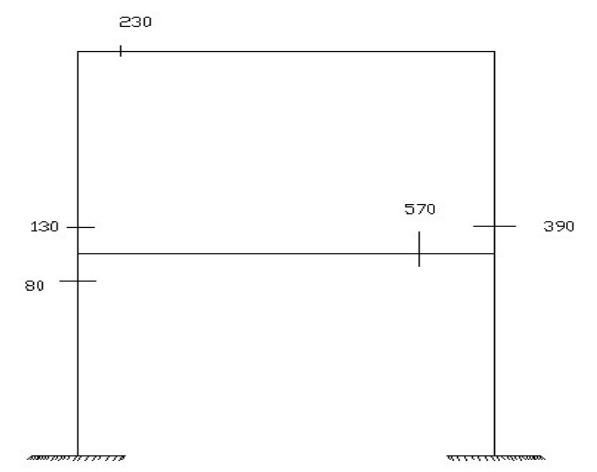

Table 1 working condition

Damage Location Damage degree

$80 \quad 0.10$

390

0.20

Fig. 1 A-span and two-storey frame model 


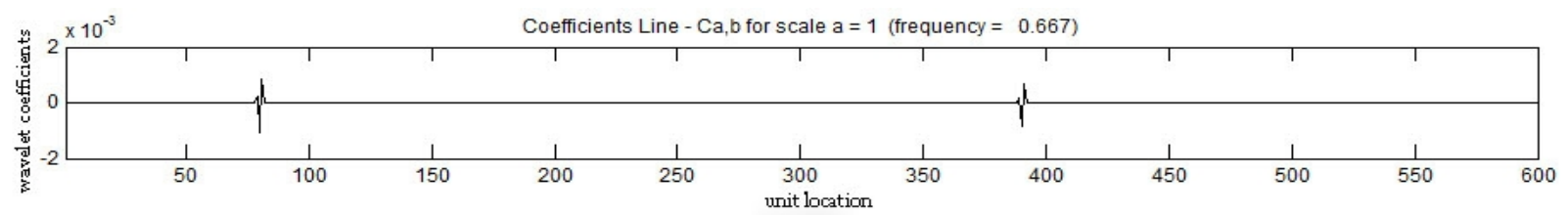

Fig. 2 Wavelet coefficient

The 80th and 390th units have obviously singular points and they are damage location. And then use RGMY to identify the degree of damage and there is the analytical graph of outcome:

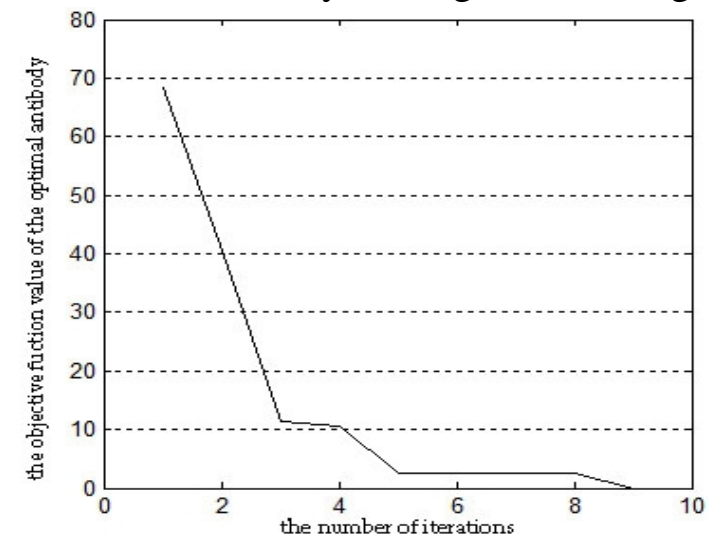

Fig. 3 Change of objective function

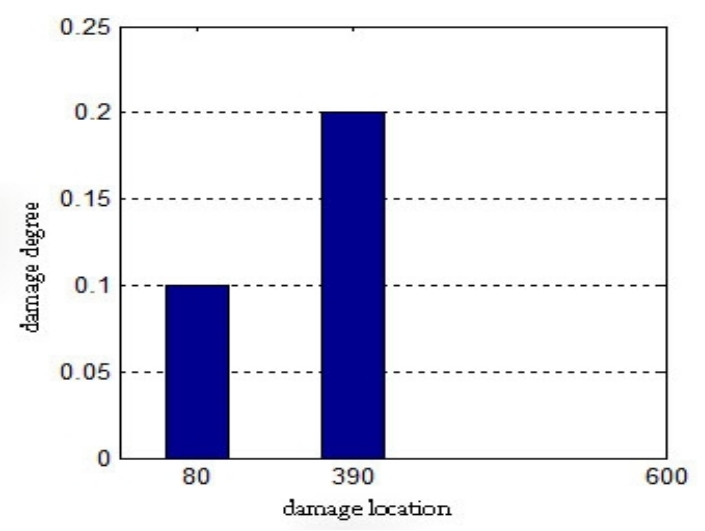

Fig. 4 Damage degree map

It can be seen from Fig. 3 and Fig. 4 that the optimal solution of the objective function is obtained when the algorithm is iterated to the ninth time, and the damage degree of the unit 80 and the unit 390 is $10 \%$ and $20 \%$, consistent with the assumptions.

\section{The Results and Conclusions}

This is the first time to combine wavelet transform and artificial immune algorithm for analysis and calculation of simple structure damage diagnosis and we call this new method the wavelet-artificial immune algorithm. The RGMY not only can identify the damage location effectively, also calculate the extent of damage accurately. Actually this paper is only applied to some simple structures, but there is no doubt that it could be applied to other complicated structures in the future.

\section{Acknowledgements}

This work was financially supported by the National Natural Science Foundation of China (513780 79).

\section{References}

[1] Hong J-C, Kim Y, et: International journal of Solids and Structures Vol. 39(2002), p. 1803

[2] Rucka M and Wilde K: Journal of Sound and Vibration Vol. 297(2006), p. 536

[3] Guan DQ, Zhong XL, Ying HW: Applied Mechanics and Materials Vol. 71-78(2011b), p. 4074

[4] Guan DQ, Ying HW, Zhong XL: Applied Mechanics and Materials Vol. 71-78(2011a), p. 4078

[5] Guan De-qing,He Tao,Tang Jun: AIIE.AIIE2016.France:Atlantis Press(2016).

[6] Bersini H and Varela FJ: Parallel Problem solving from Nature Vol. 496 (1990), p. 343

[7] Zhou Y, Tang S , et: Journal of Shenyang Jianzhu University Vol. 945-949 (2013), p. 378

[8] Guo XY, ZL: Journal of Civil Architectural \& Environmental Engineerin Vol. 34(2012), p. 7 